\title{
Experiences with data processing to separate the images in pancreatic scanning
}

D. CHARLESWORTH, H. J. TESTA, B. R. PULLAN, AND

H. BRUCE TORRANCE

From the Department of Gastroenterology, Manchester Royal Infirmary

SUMMARY In many subjects, overlap of the image of the pancreas by the liver image makes clear delineation of the pancreas uncertain. A dual isotope technique was evolved with a ratio substract system. Experiments in vitro with a phantom liver and pancreas successfully erased the liver image from the final printout. In vivo, however, the results were in many cases unsatisfactory. A detailed statistical analysis of the data has provided an explanation for these differences.

In $75 \%$ of a series of 117 pancreatic scans the pancreas was clearly visualized. A percentage in close agreement with that of other authors (Blau and Bender, 1962; Burke and Goldstein, 1964; and Bouchier, 1967). Other papers have reported a higher percentage of scans with clear visualization of the pancreas (Kakehi, Tateno, Uchiyama, and Tsuchiya, 1967) but the method of these authors has proved less effective in our hands. Our failure, in common with others (Burdine and Haynie,1965; Sodee, Renerts, Hill, and DiStefano, 1965; Rodríguez-Antúnez, 1966; and RodríguezAntúnez, Filson, Sullivan, and Brown, 1966), to improve the selectivity of selenomethionine for the pancreas, allied to the fact that optical separation methods are unsatisfactory, led us to investigate a dual isotope technique in an attempt to separate the pancreatic image from the combined liver and pancreas scan.

A dual isotope technique with computer separation of the pancreatic image has advantages of being unaffected by the duration of the scan and movement of the patient. An additional advantage is that only one scan is required.

Ratio subtract devices to detect selective localization of an isotope, have been described, the result being displayed either as a photodot recording or numerically (Ben-Porath, Clayton, and Kaplan, 1966; Kaplan, Ben-Porath, Fink, Clayton, and Jacobson, 1966; Ben-Porath, Clayton, and Kaplan, 1967; Burn, Cottrall, and Field, 1967; and Blanquet, Beck, Fleury, and Pallais, 1968. Ben-Porath et al (1967) used a method of electronic separation of the information from two pulse height analysers in a modified magnascanner. Blanquet et al (1968) used an Anger camera and two isotopes, ${ }^{75} \mathrm{Se}$ and ${ }^{198} \mathrm{Au}$. Kaplan et al (1966) used ${ }^{75}$ selenomethionine and colloidal gold, ${ }^{198} \mathrm{Au}$.

In our experiments with this type of separation we chose ${ }^{9} \mathrm{~m}$ technetium labelled sulphur colloid as a liver-seeking isotope. The advantages of technetium are its short physical half-life of six hours, and the absence of $\beta$-radiation, both of which contribute to a reduction in the dose of radiation given to the patient. The standard dose of $1 \mathrm{mCi}$ of ${ }^{99 \mathrm{~m}} \mathrm{Tc}$ sulphur colloid used for liver scanning produces high count rates and thus reduces the statistical uncertainties for every point of the liver scan. Combination with ${ }^{75} \mathrm{Se}$ methionine allows a normal selenium liver and pancreas scan to be recorded simultaneously without interference from the liver seeking isotope. 
THEORY OF DUAL ISOTOPE SCANNING WITH COMPUTER SEPARATION

Liver

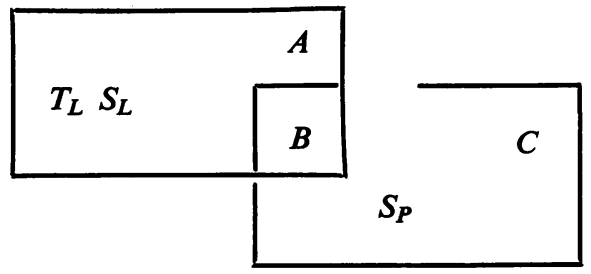

Pancreas

$T_{L}$ is ${ }^{99}$ technetium in the liver.

$S_{L}$ is ${ }^{75}$ selenium in the liver.

$S_{P}$ is ${ }^{75}$ selenium in the pancreas.

$\eta_{y}^{x}$ is the efficiency for counting isotope $x$ in the detector channel for isotope $y$

In area $A$ total count/unit of time in technetium channel is $C_{T}=T_{L \eta T}^{T}+S_{L \eta_{T}^{s}}$

Total count/unit of time in the selenium channel is $C_{S}=S_{L \eta_{s}^{s}}$

Inarea $B C_{T}=T_{L \eta_{T}^{T}}+S_{L \eta_{T}^{t}}+S_{P \eta_{T}}$ $C_{S}=\left(S_{P}+S_{L}\right)_{\eta_{s}^{s}}$

In area $C C_{T}=0$

$$
C_{S}=S_{P \eta_{s}^{s}}
$$

Defining, from region $A$, a normalizing factor $K=\frac{S_{L \eta_{s}}^{s}}{T_{L \eta_{T}^{T}}+S_{L \eta_{T}^{s}}^{s}}$

The excess activity in area $B=C_{S}-K C_{T}$

$$
=S_{P \eta_{s}}^{s}+S_{L \eta_{s}^{s}}-K T_{L \eta_{T}^{T}}-K S P_{\eta T}{ }^{s}-K S_{L_{\eta}}{ }_{s}
$$

as $K T_{L \eta_{T}^{T}}+K S L \eta_{T}^{s}=S L_{\eta_{s}^{s}}$

The excess activity in area $B=S_{P \eta_{s}^{s}}-K S_{P \eta_{T}^{s}}$

$$
\begin{aligned}
& =S_{P \eta_{s}^{s}}-K_{\eta_{T}} \\
& =K_{1} S_{P}
\end{aligned}
$$

With this simple normalization the excess is proportional to $S_{P}$

In area $C$ any excess activity $=S_{P \eta_{s}^{s}}=K_{2} S_{P}$

Thus outside the liver there is a different constant of proportionality with this method of normalization.

$$
\frac{K_{1}}{K_{2}}=\frac{\eta_{s}^{s}-K_{\eta_{T}^{s}}}{\eta_{s}^{s}}=1-\frac{K_{\eta_{T}^{s}}}{\eta_{s}^{s}}
$$

Because of this the pancreas will appear to change in activity as we pass over the edge of the liver.

The magnitude of this effect in scan practice can be seen by substituting a typical value of $K=0 \cdot 15$ and that of $\frac{\eta_{T}^{s}}{\eta_{s}^{s}}=0.8$ thus $\frac{K_{1}}{K_{2}}=0.9$. This $10 \%$ change in activity will not make the system un- usable and is similar to the discrepancy which occurs in the work of others who used ratio subtraction devices.

\section{Materials and Method}

EXPERIMENT 1

A liver phantom was made by encasing a human cadaver liver in fibreglass and subsequently removing the liver after the case had hardened. A plastic tube, 7 in. in length by $1 \frac{1}{2}$ in. in diameter, was used as a pancreas phantom. The liver was filled with water containing sufficient ${ }^{99 \mathrm{~m}}$ technetium and ${ }^{75} \mathrm{Se}$ lenomethionine to give rates of 20,000 counts/minute and 3,000 counts/ minute respectively in the two channels. These figures are comparable to count rates encountered in scanning patients. A similar rate of 3,000 counts/minute in the area of the pancreas phantom not overlapped by the liver, was obtained by adding ${ }^{75}$ selenium methionine to the solution filling the pancreas phantom.

A conventional Picker $\mathrm{V}$ magnascanner was used, to which had been added a second pulse height analyzer. The count rates from both channels were recorded on magnetic tape. The position of the head of the scanner was recorded simultaneously on a third channel. This was achieved by fitting the carrying arm with a strip of metal slotted at regular intervals. A signal was produced each time a slot passed between a light source and a photoelectric cell.

The data from the ${ }^{75} \mathrm{Se}$ and ${ }^{90 \mathrm{~m}} \mathrm{Tc}$ channels were punched on separate computer tapes. The ${ }^{99 \mathrm{~m} T \mathrm{Tc}}$ channel data (liver scan) was read into the computer and stored as an array of numbers multiplied by the normalizing factor. The ${ }^{75} \mathrm{Se}$ channel data (liver and pancreas scan) was read in as a second array of numbers. Both scans were printed out as density maps, using symbols with different apparent blackness on the line printers. The liver array was then subtracted from the liver and pancreas array, the result being again printed out as a density map. The statistical significance of each point was calculated and a fourth density map was printed, in which each point was assigned a density according to its significance. The computer determined the point of highest significance and divided the range between zero and this value into eight intervals, each of which had a density symbol.

EXPERIMENT 2

Ten normal volunteers were given an intravenous injection of $200 \mu \mathrm{Ci}$ of ${ }^{75}$ selenomethionine and $1 \mathrm{mCi}$ of ${ }^{99} \mathrm{~m} \mathrm{Tc}$ sulphur colloid. The site of maximum liver activity was found, using the scanner head as a probe, the ratio of ${ }^{75} \mathrm{Se}$ to ${ }^{99 \mathrm{~m}} \mathrm{Tc}$ was recorded at this site. A complete liver and pancreas scan was performed starting 10 minutes 


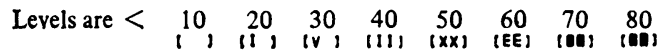

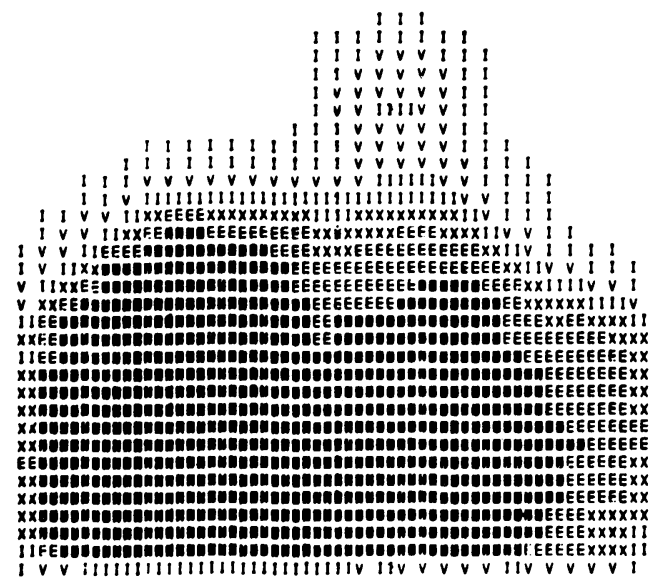

Fig. 1 A density graph of combined liver and pancreas phantoms. The scale shows the density symbols and the count rates to which these correspond.

after the injection. The conditions for scanning and the sequence of movements of the head were identical in all cases. The data from the two pulse height analyzers was processed as in the first experiment.

\section{EXPERIMENT 3}

A flat plastic jig was made to fit horizontally over a patient lying supine. The surface was marked in a regular pattern of $\frac{1}{2}$ in. squares. Eight points were numbered on this jig lying in an even distribution over the liver of the patient.

Ten minutes after an injection of $200 \mu \mathrm{Ci}$ of selenomethionine and ${ }^{99 \mathrm{~m}} \mathrm{Tc}, 1 \mathrm{mCi}$ of sulphur colloid, the count rates at each of these points in strict numerical order was recorded for 40 seconds at each part. A line-by-line liver and pancreas scan was carried out, moving the scanner head at $\frac{1}{2}$ in. intervals and counting for 10 seconds at each point. At the end of each alternate line the set pattern of counts over the liver was repeated.

\section{Results}

EXPERIMENT 1 (PHANTOM EXPERIMENTS)

Figure 1 shows the density graph of the combined liver and pancreas phantoms. The head of the pancreas can be seen projecting from the upper border of the graph. Figure 2 is the density graph of the separated pancreatic image, showing sharp delineation of the pancreas and almost complete eradication of the liver, and Figure 3 is the density graph obtained by calculating the statistical significance of each point in Figure 2.
Pancreas:- Student's T Distribution

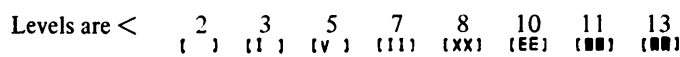

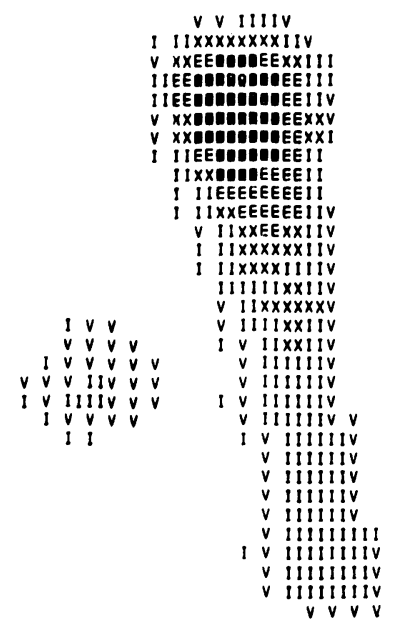

Fig. 2 A density graph of the separated pancreas, scale indicating density symbol and related count rate.

\section{Pancreas}

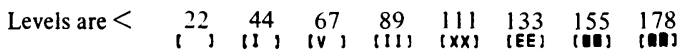

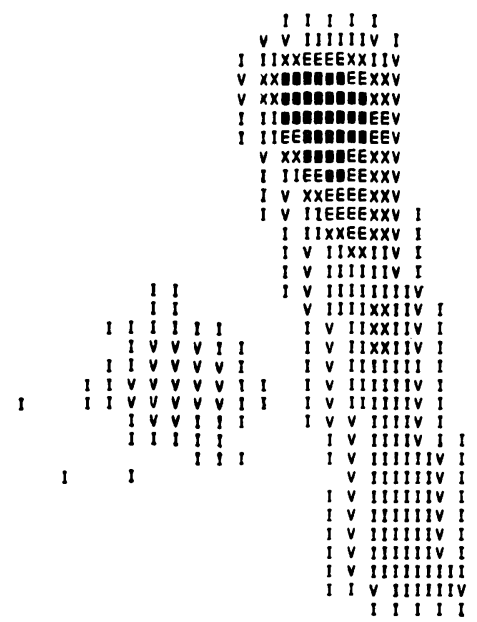

Fig. $3 A$ density graph of pancreas Student's $t$ distribution scale shows density symbol and related order of significance. 
EXPERIMENT 2 (NORMAL VOLUNTEERS)

Complete separation of the pancreas from an overlying liver was achieved in only two out of eight cases. Changing the normalizing factor in each of the other scans did not improve the image (Figures 4 and 5). The statistical significance of each point on Figs. 4 and 5 was calculated, and Figs. 6 and 7 show a separated liver and pancreas in which each point was assigned a density according to its significance.

\section{EXPERIMENT 3}

The count rates from the fixed pattern of points over the liver was recorded, and the exact time of each repeat-count noted. The count rates from the liver and pancreas scan were recorded as a matrix of numbers, which were corrected for background, and the crosstalk effect of ${ }^{75} \mathrm{Se}$ in the ${ }^{99 \mathrm{~m}} \mathrm{Tc}$ channel.

The ratio of ${ }^{99 \mathrm{~m}} \mathrm{Tc}$ to ${ }^{75} \mathrm{Se}$ was calculated over the liver, and these ratios were plotted as a graph (Fig. 8), which shows that there is some variation in this ratio in respect to time. The ratio was $0 \cdot 18 \pm 0 \cdot 01$. The corrected ${ }^{99 \mathrm{~m}} \mathrm{Tc}$ count at each point on the scan was multiplied by this figure and the result subtracted from the selenium count. The final ${ }^{75} \mathrm{Se}$ figures were plotted as a series of histograms (Fig. 9) and reproduced as a defocused photograph (Figure 10). Figure 11

\section{Averaged Liver and Pancreas}

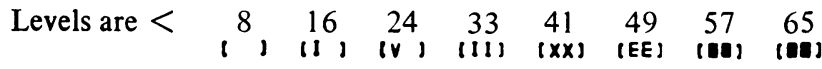

\section{1}

$\begin{array}{llllllllllllllllllllllllllllllllllll}1 & 1 & 1 & 1 & 1 & 1 & 1 & 1 & 1 & 1 & 1 & 1 & 1 & 1 & 1 & 1 & 1 & 1 & 0 & 1 & 1 & 1 & 1 & 1 & 1 & 1 & 1 & 1 & 1 & 1 & 1 & 1\end{array}$

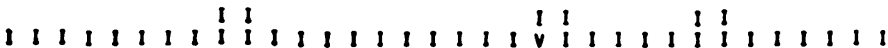

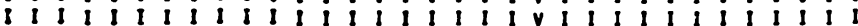

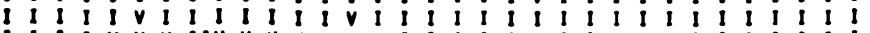

i i i i v v vituvivava

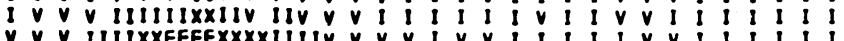

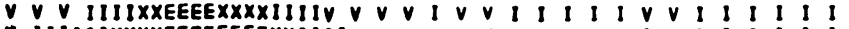

I IIIIIXXXXEEEEEEEEXXIIIV v v v v v v v v v v I v i i i i i 11

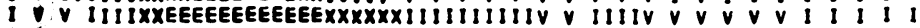

i MiIXXEEOAEEE

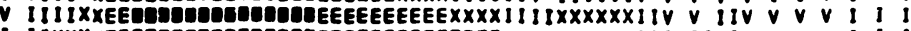

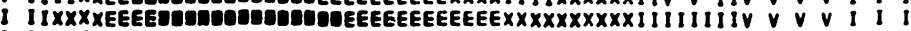

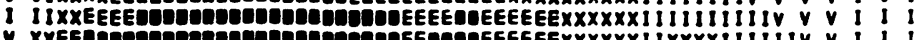

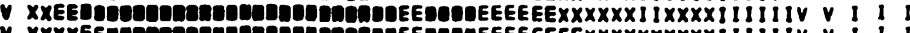

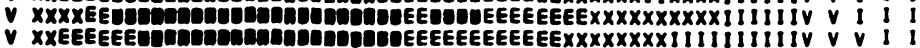

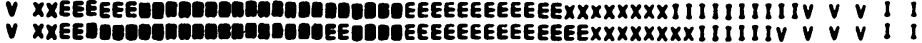

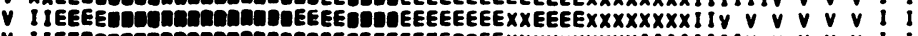

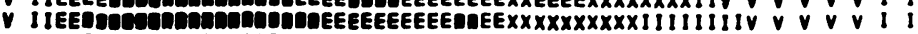

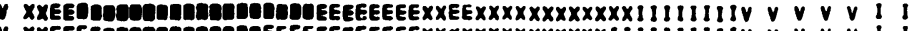

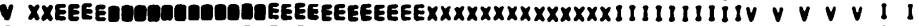

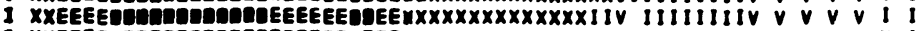

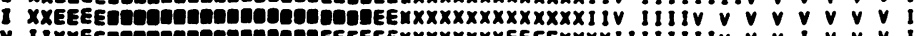

V IIXXEEG

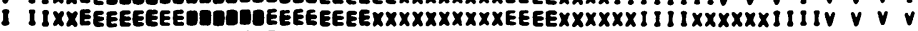

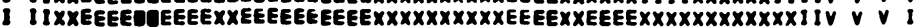

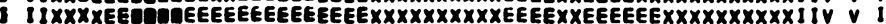

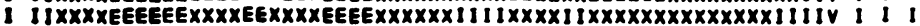

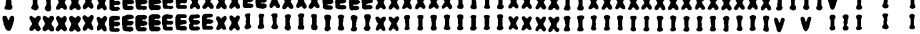

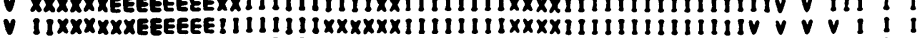

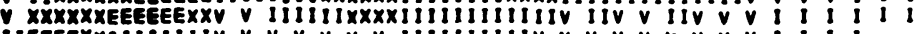

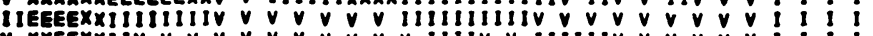

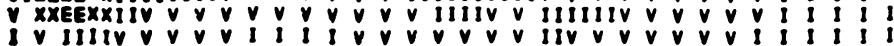

Fig. 4 Typical density map of the liver and pancreas obtained from a normal volunteer. shows clearly the edge of the liver and the excess counts over the pancreas.

Using the figures obtained from the most active areas of the liver and pancreas, the statistics of scanning can be worked out, assuming that the liver overlies an active area of the pancreas, and that the distribution of activity in both liver and pancreas is uniform.

\begin{tabular}{|lll}
$\begin{array}{l}T_{L}=2,500 \\
S_{L}=400\end{array}$ & $A$ \\
& $\begin{array}{l}\text { LIVER } \\
S=600 \\
T=2,500\end{array}$ \\
\hline \multicolumn{2}{|c|}{$S_{P}=200$} \\
\end{tabular}

Excess activity in

area $B$

$$
=C_{S}-K C_{T}
$$

Then selenium in

$$
\begin{aligned}
\text { the overlap area } & =600 \pm 25-(2,500 \pm \\
& 50) \frac{400}{2,500} \\
& =600 \pm 25-400 \pm 8 \\
& =200 \pm 33
\end{aligned}
$$

This is very significant (difference $=6 \mathrm{SD}$ ).

In a real scan the collimator stops over each resolution area for one second.

$$
\text { Selenium in area } \begin{aligned}
B & =60 \pm 8-(250 \pm 16) \frac{40}{250} \\
& =60 \pm 8-40 \pm 3 \\
& =20 \pm 11
\end{aligned}
$$

This is not very highly significant and represents the best situation, as the figures used in the calculation are representative of the most easily separated areas.

\section{Discussion}

The result of our first experiment, using a pancreas and liver phantom, was encouraging. The outline of the 'pancreas' was clear, and the 'liver' was erased. In only two of eight dual isotope scans in human volunteers were similarly clear images obtained, in one of which there was a good separation of the two images on the photodot recording.

The rationale of this method of separation is based upon the premise that the ratio of ${ }^{99 \mathrm{~m}}$ technetium to ${ }^{75}$ selenium is constant in the liver. An explanation as to why we could not separate the images clearly is that this ratio varies with time or from one area to another within the liver. The usual time to complete a liver and pancreas scan is approximately 30 minutes. That the ratio should vary with time seems likely from our 


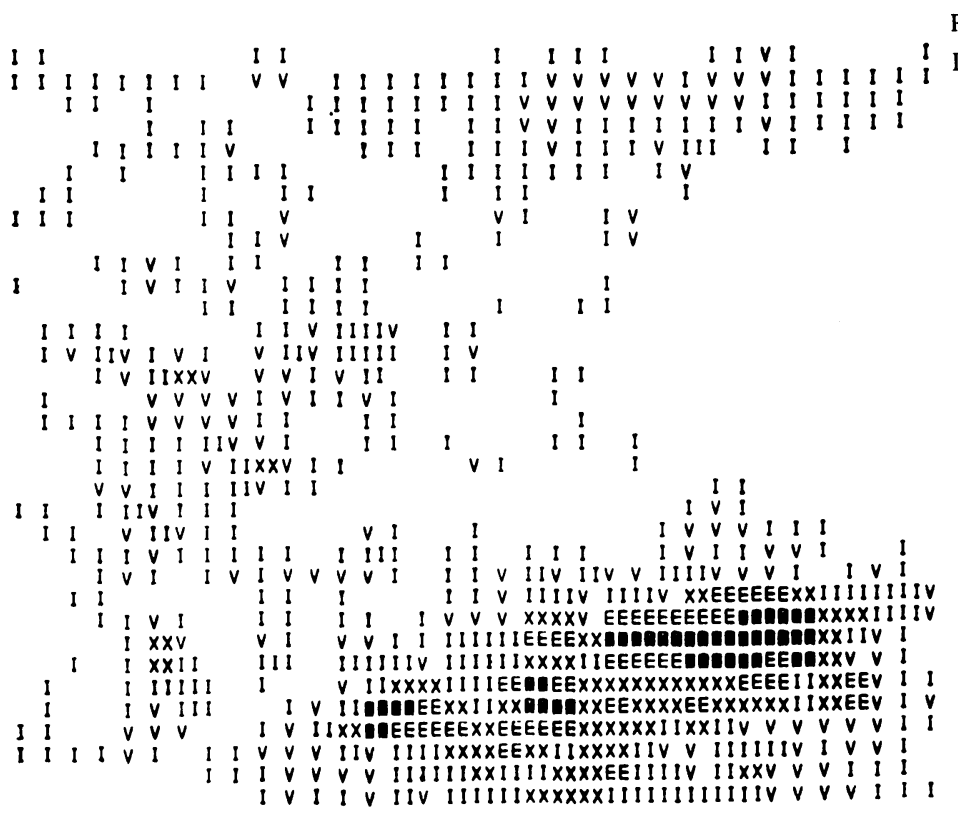

Fig. 5 Separated pancreatic image, obtained from subtraction of the data in Figure 4.

No.of Rows now $=35 \quad$ No. of Cols now $=35$

Averaged Liver

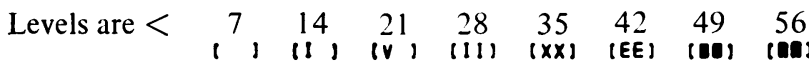

111111111111

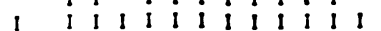

$11^{1} 1$

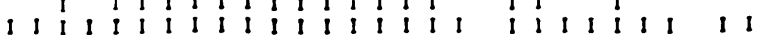

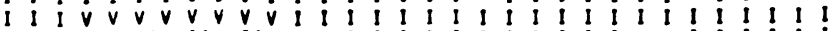

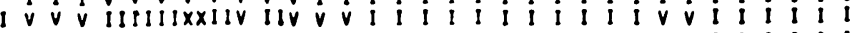

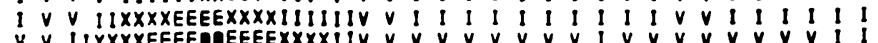

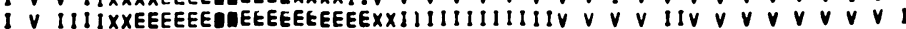

1 IXXXXEEEREA

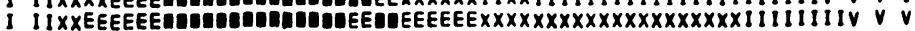

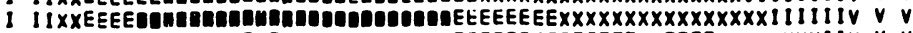

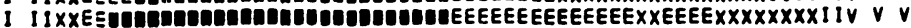

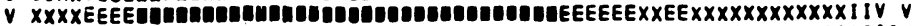

v $\times$ XEEEE

$\checkmark$ XXEEOMO0000

$\checkmark$ XXEEO

$\checkmark$ XXEEQ

$v$ $x \times E E$ Bou

$v$ XXEE

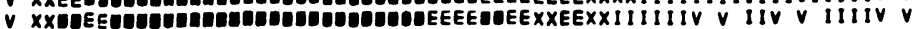

$v$ XXEEEEEE

v XXEEEEP

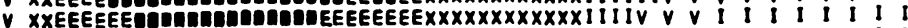

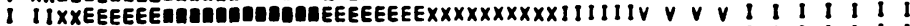

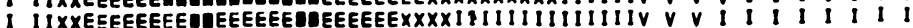

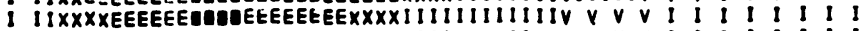

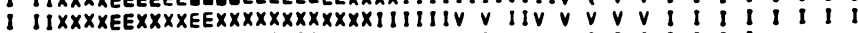

$v$ XXEEEEEEXXXXEEXXIIIIIIIV $v v v v$ i v $v i$ I 1 i 1

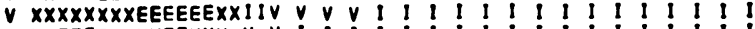

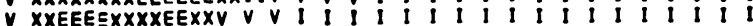

$\checkmark$ EEEEXXIIIIIIIIV 11

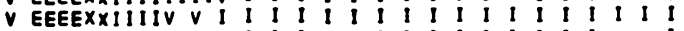

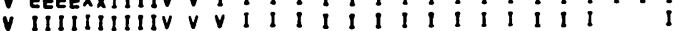

Fig. 6 Separated liver image after calculation of the statistical significance of each point.
Pancreas:- Student's T Distribution

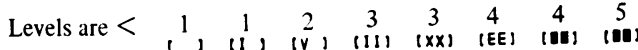

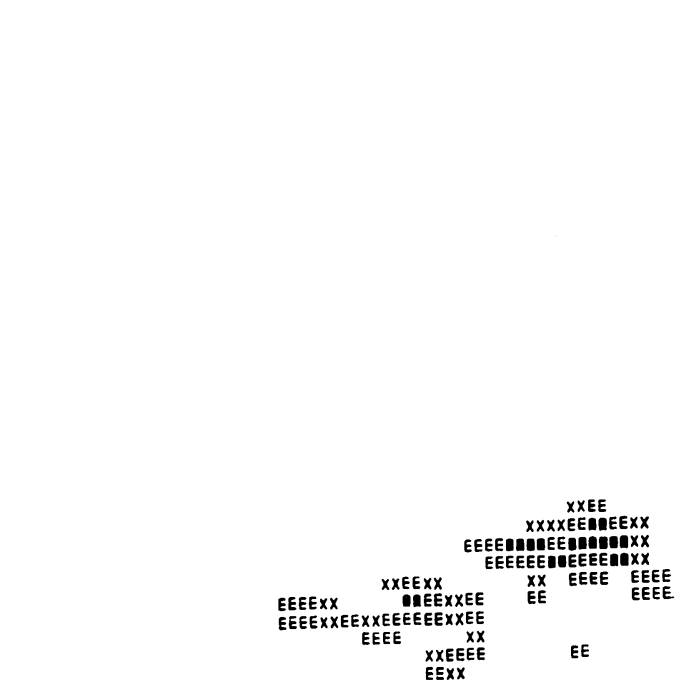

Fig. 7 Separated pancreas image after calculation of the statistical significance of each point.

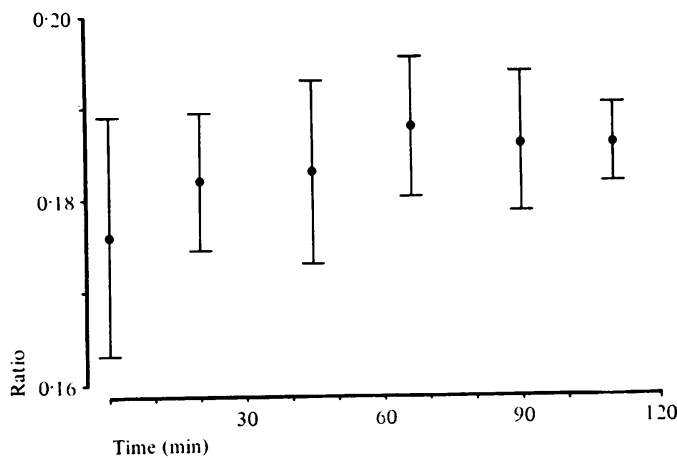

Fig. 8 The ${ }^{99 \mathrm{~m}} \mathrm{Tc}$ to ${ }^{75} \mathrm{Se}$ ratio in the liver, and its variation with time during the course of experiment 3. Each point represents the mean of the eight ratios measured over the liver. The vertical lines represent the standard deviation at each point.

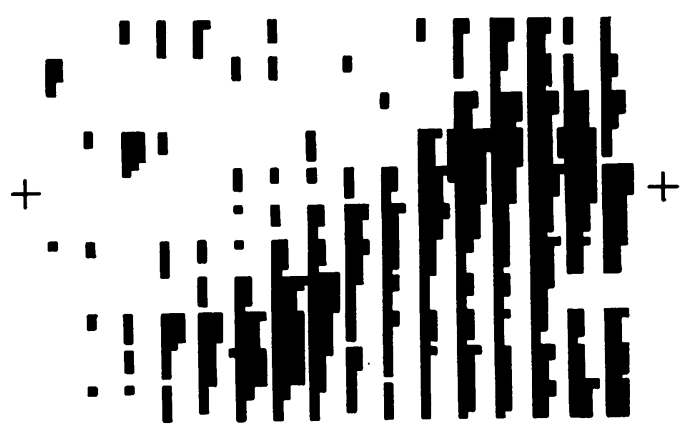

Fig. 9 The excess ${ }^{75} \mathrm{Se}$ counts at each point on the hand scan (experiment 3) reproduced as a series of linear histograms. The line between the two pluses $(+)$ is the line from which the values used in Fig. 11 were obtained. 


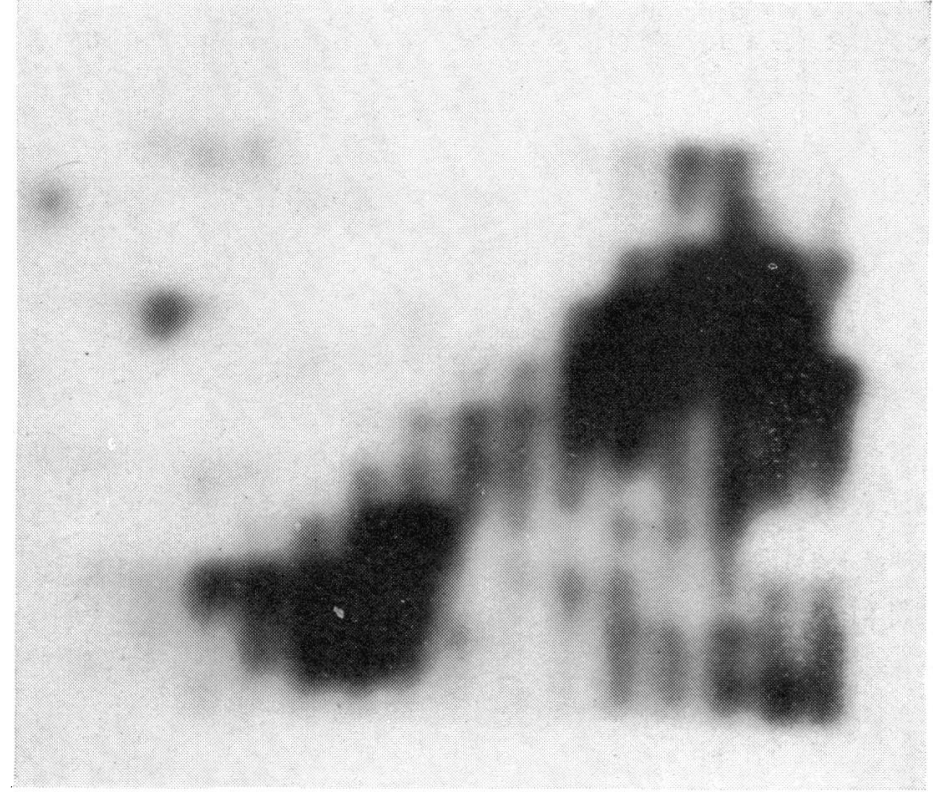

Fig. 10 A defocused photograph of Figure 9.

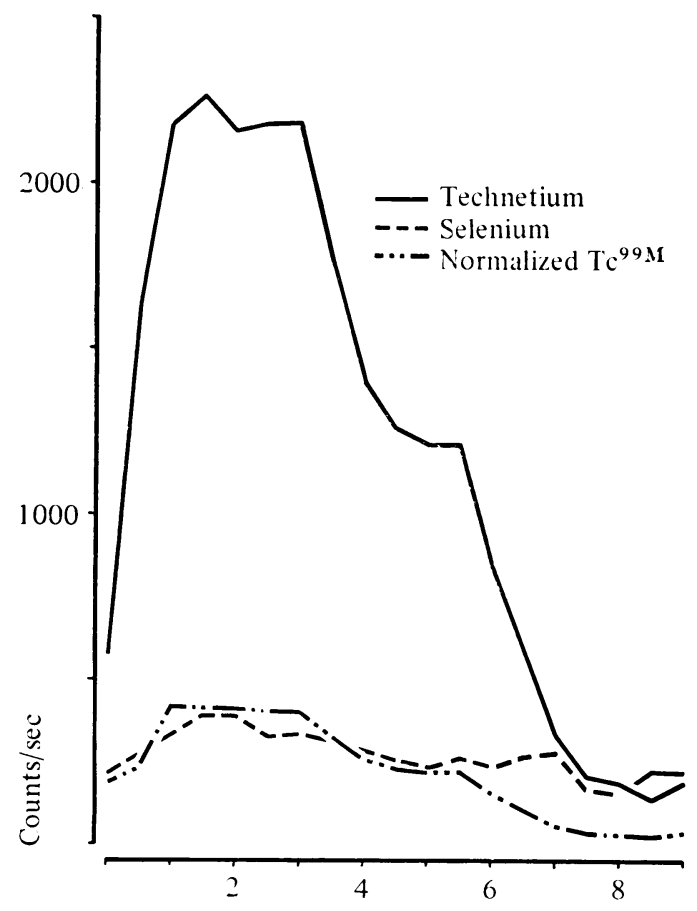

Position (inches)

Fig. 11 Graph of counts at each point in the line between the two pluses on Fig. 9, against position along this line. The solid line shows clearly the sharp drop in counts at the liver edge. The selenium line shows little variation from side to side, but exceeds the technetium line and lies well below the selenium line in the region of the pancreas. From 6 inches to 9 inches.

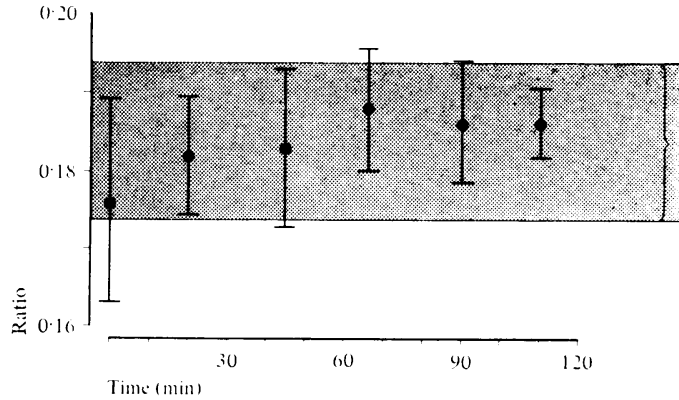

Fig. 12 A reproduction of Figure 8. The stippled area represents a value of $K=0.02$, the upper limit of acceptable variation.

experiments on the metabolism of selenomethionine, which demonstrated that selenomethionine is rapidly metabolized in the liver and returned to the blood incorporated in the plasma proteins.

From our third experiment (Fig. 8) it can be seen that the scan ratio of ${ }^{99 \mathrm{mTc}}$ to ${ }^{75} \mathrm{Se}$ did change with time, and although small the change is statistically significant. The computed ${ }^{75}$ selenium activity in the liver in the overlap area is $K C_{\mathrm{T}}$ where $K$ is the normalizing factor and $C_{\mathrm{T}}$ the count rate in the technetium channel. If $K$ varies by an amount $T K$, on subtraction, this will give areas of apparent pancreas activity of the order of $K T$. In the third experiment the area of least activity in the pancreas was 100 counts in 10 seconds. If we assume that in order to obtain good subtraction images no area in the region of the liver, after subtraction, exceeds half this value.

$$
\text { Then } \quad \begin{aligned}
K T & \frac{100}{2} \\
\text { as } \quad T & =2,500 \\
\text { Then } \quad K & =\frac{100}{2 \times 2,500} \\
& =0.02
\end{aligned}
$$

Taking a value of $K$ as 0.02 as the upper limit in the acceptable variation in ratio, it can be seen by superimposing this value on Fig. 8 that the variations in ratio from point to point, over the liver, are of this magnitude (see Figure 12). Consequently areas in the region of the liver, which are comparable in activity to parts of the pancreas, are to be expected after subtraction.

In a human scan, the statistical fluctuations are considerably greater, thus anomalous areas of pancreas are likely to appear. The figures used in obtaining the ratios were all taken from an area over the body of the liver. It is possible that even greater variation would be encountered over the edge of the liver where the overlap occurs.

An explanation of the expertise developed, on the part of the pancreatic scan interpreter is that 
the assessment of data is carried out in an entirely different way from that employed by the computer. The computer was programmed to produce a density map showing all points with a probability above a fixed minimum of 2 standard deviations. Observer scan interpretation is a process of pattern recognition. Probably this is a process in which the observer subconsciously superimposes a preconceived pancreatic image on the scan, fitting dots of equal density into this pattern. Variations are thus detected as aberrations on the scan, not fitting one of the acceptably normal patterns.

\section{CONCLUSIONS}

A dual isotope technique with a ratio subtract device can be used to separate the pancreas from a liver plus pancreas scan; but so far the technique has no advantage to offer over photodot recording.

In the absence of a compound with a higher selectivity for the pancreas than selenomethionine, interpretation of the photodot recording by an experienced observer is at present as good as any of the ratio methods as yet published.
References

Ben-Porath, M., Clayton, G., and Kaplan, E. (1966). Selective visualization of the pancreas by subtractive double-radioisotope scanning technique. Trans. Amer. nucl. Soc., 9, 76-78.

Ben-Porath, M., Clayton, G. D., and Kaplan, E. (1967). Modification of a multi-isotope color scanner for multi-purpose scanning. J. nucl. Med., 8, 411-425.

Blanquet, P. C., Beck, C. R., Fleury, J., and Pallais, C. J. (1968) Pancreas scanning. ${ }^{75} \mathrm{Se}$ selenomethionine and ${ }^{108} \mathrm{Au}$ colloidal gold using digital data processing techniques. $J$. nucl. Med., 9, 486-488.

Blau, M., and Bender, M. A. (1962). ${ }^{75}$ Se-selenomethionine for visualization of the pancreas by isotope scanning. Radiology, 78, 974.

Bouchier, I. A. D. (1967). Pancreatic scanning. Gut, 8, 421-422

Burdine, J. A., and Haynie, T. P. (1965). Diagnosis of pancreatic carcinoma by photoscanning. J. Amer. med. Ass., 194, 979-983.

Burke, G., and Goldstein, M. S. (1964). Radioisotope photoscanning in the diagnosis of pancreatic disease. Amer. J. Roentgenol., 92, 1156-1161.

Burn, G. P., Cottrall, M. F., and Field, E. O. (1967). A ratiosubtract device for detecting selective localisation of isotopes in clinical scintiscanning. Brit. J. Radiol., 40, 62-65.

Kakehi, H., Tateno, Y., Uchiyama, G., and Tsuchiya, S. (1967). Radioisotope scanning of pancreas carcinoma. (Abstr.) J. nucl. Med., 8, 387-388.

Kaplan, E., Ben-Porath, M., Fink, S., Clayton, G. D., and Jacobson, B. (1966). Elimination of liver interference from the selenomethionine pancreas scan. J. nucl. Med., 7 , 807-816.

Rodriguez-Antúnez, A., Filson, E. J., Sullivan, B. H., Jr., and Brown, C. H. (1966). Photoscanning in diagnosis of carcinoma of the pancreas. Ann. intern. Med., 65, 730-737.

Sodee, D. B., Renerts, L., Hill, G., and Di Stefano, B. (1965). Dosimetry of selenomethionine ${ }^{75} \mathrm{Se}$ for pancreatic scan ning. Nucleonics, 23, 78. 